\title{
The effect of social and existential aspects during end of life
}

\section{care}

\author{
Patricia Dobríková ${ }^{1}$, Jana Macková ${ }^{1}$, Lukáš Pavelek ${ }^{1}$, Layla K AlTurabi ${ }^{2}$, Aimee Miller ${ }^{2}$ and Daniel J West ${ }^{2 *}$ \\ ${ }^{1}$ Department of Healthcare and Social Work, Trnava University, Trnava, Slovakia \\ ${ }^{2}$ Department of Health Administration and Human Resources, University of Scranton, PA, USA
}

\begin{abstract}
Objective: This study discusses the social and existential aspects affecting patients during end of life care. It examines the impact of specific indicators on patient perception of the purpose and meaning of life.

Methods: This study was conducted directly on the terminally ill patients receiving palliative care $(\mathrm{N}=32)$. A standardized Logo-test was used in the research to measure specific indicators of care.

Results: There was a significant correlation between the level of satisfaction with social support from relatives of the dying client and the degree of meaningfulness of his or her life.

Significance of results: The results of the study suggest that social support, awareness, and meeting patients' needs are among indicators that significantly affect patients' meaningfulness of life. Extending the care given to terminally ill patients beyond pain management and symptom control to include the treatment of other problems associated with the psychosocial, existential, and spiritual status appears to have efficacy.
\end{abstract}

\section{Introduction}

Improving the quality of life and the meaningfulness of life perceived by the terminally ill patients is becoming prominent in palliative care settings. The World Health Organization (WHO) defines palliative care as "an approach that improves the quality of life of patients and their families facing the problem associated with lifethreatening illness, through the prevention and relief of suffering by means of early identification and impeccable assessment and treatment of pain and other problems, physical, psychosocial and spiritual" [1]. Quality of life is achieved when ultimate physical, psychological, social, and spiritual well-being is achieved. It is to some extent related to meaning and life fulfillment. The dying experience induces a number of changes and reactions in the patients and their families. Only patients suffering from a terminal illness understand the experience of living with such an illness. The purpose of this study is to examine the effect and understand the importance of social and existential well-being at end of life by examining the impact of three specific indicators on patient perception of the purpose and meaning of life: source of meaning and values, expression of existential frustration, and perception of meaningfulness.

\section{Social aspects of terminally ill patients}

Incurable illnesses change the social status of the patient. Besides pain, and other devastating symptoms and complications, patients may suffer from the undesirable effects of the disease which affects the patient's appearance; the loss of social, professional, and familial roles; the ability to remain independent and function normally, and most importantly the perception of the future. According to Sherbourne and Stewart [2], social support serves various dimensions including (1) emotional support which is defined as being empathetic and understanding, having positive affect, and encouraging the expression of feelings; (2) providing aid and assistance such as transportation, financial and/or housekeeping help is considered as instrumental support; (3) informational support involves offering information, guidance, and advice; and (4) affectionate support which consists of having someone who expresses love and affection. Helgeson [3] suggests that social relationships put patients in a better mood and provide them with a sense of identity and companionship. Social support might influence patients' quality of life and meaningfulness of life by helping them cope more effectively with their sufferings and making them feel valued, loved, and cared for. Moreover, Schwartz and Frohner [4] found that the more social support a patient perceived, the less pain suffered, and the better he/she assessed general health and well-being. In a study conducted on terminally ill adult patients diagnosed with cancer, to understand the meaning of social well-being at the end of life, Prince-Paul [5] found that all of the participants in the study identified the need to be surrounded by family and participate in social activities. This, in turn, provided patients with a reason to live and a purpose to stay involved and live while they were dying. Similarly, Mikulincer, Florian, and Hirschberger [6] postulate that close social relationships that transcend physical death may provide a protection

Correspondence to: Daniel J West, Jr, PhD, FACHE, Department of Health Administration and Human Resources, University of Scranton, 417 McGurrin Hall, Jefferson Avenue, Scranton, PA 18510, USA; Tel: 570- 941-4126; E-mail: westd1@scranton.edu

Key words: social aspects, existential aspects, meaning of life, terminally ill, palliative care

Received: March 10, 2016; Accepted: April 14, 2016; Published: April 18, 2016 
that enables the patient to better cope with the reality of death. They suggest that this close relationship promotes self-preservation, help patients with death concerns, and assist them in realizing the meaning and value of their lives.

\section{Existential aspects of terminally ill patients}

Issues regarding existentialism are a central concept in palliative care. Existentialism at the end of life can be defined as "hopelessness, futility, meaninglessness, disappointment, remorse, death anxiety, and disruption of personal identity" $[7,8]$. Existential philosophers described existential issues to be related primarily to four basic aspects with which each terminally ill patient must struggle: meaninglessness, loss of freedom, existential isolation, and death anxiety [9]. More precisely, these different issues relate to patient end of life situational experiences which can be summarized as "(1) the experience of dependency inability and restrictions on freedom, (2) the experience of meaning or lack of meaning, (3) the struggle for daily life and survival, (4) relations with close relatives and significant others, (5) communications with others, (6) thoughts about the future, in particular, the dying process, (7) concerns about the family, (8) feelings of guilt for former actions and present life situations, (9) thoughts about God and life after death, and (10) personal suffering" [10]. Frankl's [11] existential analysis and logo therapy strengthen the concept of human participation in addressing adverse social situations, especially in crisis.

Blinderman and Cherny [12] suggest that "existential issues may be mitigated by a strong framework of palliative measures, good family support, effective coping strategies, and religious belief systems." A study conducted by Moadel et al. [13] on identifying cancer patient needs in a palliative setting found that patients overcame fears, found hope, meaning of life, and spiritual resources.

\section{Methods}

\section{Research objectives}

Three basic research questions were established for the research study. The points of interest were as follows: whether there is a relationship between social support and the meaningfulness of life of the dying client; whether the satisfaction with the fulfillment of their bio-psycho-socio-spiritual needs affects the meaning of life of the dying; and finally, whether there is a link between the client's level of awareness and their perception of the meaning of life.

\section{Eligibility and exclusion criteria}

The answers were sought with dying patients who were hospitalized in a hospice for at least five days and the physician's prognosis of their life expectancy was less than 1 month.

The following are some of the reasons for which several clients did not participate in the study.

1. Client was in a coma (18 clients, equals 9.63 per cent).

2. Client was unable to answer all of the research questions due to complete exhaustion $(28,14.97)$.

3. Client was unable to communicate due to organic brain damage under the influence of metastases $(56,29.95)$.

4. Client was in death agony $(11,5.88)$.

5. Client was prevented from participating in research by disturbances of sensory functions $(19,10.16)$.
6. Client was unable to finish the research because of a sudden deterioration of health $(7,3.74)$.

7. Client was mentally unfit to participate in the research $(16,8.56)$.

\section{Participants}

During the visits that were part of the research process, permissions for research were granted by two Slovak hospices. At the time of the visits, which took place from May 2013 to May 2014, a total of 187 hospital clients were hospitalized in these facilities. The number of those physically and mentally fit to answer the questions, totaled 32 . The research sample thus consists of 18 men and 14 women aged 35 to 92 , with a mean age of 76.9. All of the clients who participated in the research were Roman Catholics, which is the majority religion in the Slovak Republic. Regarding the health problems in the sample of patients, the most frequently found was cancer diagnosis (24 clients), less frequently neurological (4 clients) and cardiovascular diagnosis (4 clients).

\section{Measures}

A custom designed questionnaire for the dying clients was used and consisted of two parts. In the first part, the basic sociodemographic data were investigated. In the second part, the questions were formulated so that the clients could express themselves in the areas of inquiry. The questions were related to the patients' perceptions of satisfaction with the positive effect of medical treatment, satisfaction with psychological support, with fulfillment of spiritual needs, the implementation of nursing care and personal hygiene, social services and counseling, communication with members of the hospice team, being informed about the progress of the care in general, and support of family members and the frequency of their visits. Based on the statistical verification of the level of research validity by means of a reliability test, we can conclude that the questions in the questionnaire are sufficiently consistent, as Cronbach $\alpha=0.86$.

Another part of the research consisted of a semi-structured interview aimed at finding out who provides the client with most the support, who visits most frequently, and who is the best partner for talking about personal problems and issues relating to the meaning of life.

In our research, a standardized Logo-Test was used. Logo-Test is one of the first instruments developed to assess meaning in life which was designed and standardized in Austria by Elizabeth Lukas. She got her Ph.D. under V.E. Frankl and for her doctoral thesis she constructed and evaluated this instrument, which has a separate scale for men and women, and has been normed in many countries. The Logo-Test includes factors contributing to the feeling of meaningfulness and symptoms resulting from a weak sense of meaning in life - symptoms of existential frustration. The instrument aims to have both research and therapeutic applicability: apart from the general sense of meaning in life of a person, the Logo-Test also attempts to provide preliminary information about specific topics to work on in a potential therapeutic process. The original German-language version of the Logo-Test or parts of it has been employed in several studies being conducted in German-speaking countries. In addition, the scale was translated into at least 15 languages and was used in several countries worldwide (USA, Mexico, Slovakia, Czech Republic, Hungary, Spain, etc.) [14-16].

Logo-Test is designed for the practice of physicians, psychologists, educators, as well as social workers and clergy. The test was divided into three main sections, each of which has been evaluated separately. 
In Section I (Source of meaning and values), clients had the option to answer "yes", "no" or refuse to answer nine questions in the field of family relationships, self-realization, profession, friends and the community.

In Section II (Expressions of existential frustration), clients were asked seven questions about going through certain experiences and their potential effects (for example helplessness, agitation, and aggression). Clients rated the occurrence of given experiences as "very often", "sometimes" or "never" to all questions.

In Section III (Perception of meaningfulness), clients were presented with three different short stories of people who in their lives did not have the opportunity to act according to their wishes, and the reality had been far removed from their life goals. Yet, each of them had somehow managed to accept his or her vocation. In this part, clients were to indicate in which story the protagonist was "happy" and in which they were likely "to be suffering". Answers have again been evaluated separately. In Part III, clients also had the option of providing their own story about their life goal and whether they had achieved what they had strived during their life.

Finally, an assessment has followed, having taken into account several factors including an important variable - the age of the client participating in the research. The results are expressed in the so-called quartiles Q1 - Q4. For the quartile Q4 the deciles D9 and D10 are further defined.

Q1: Very high level of "sense of fulfillment"

Q2 + Q3: Medium level of "sense of fulfillment"

Q4 (D9 + D10): Impaired "sense of fulfillment"

D9: Low "sense of fulfillment" - Existential frustration

D10: Very low "sense of fulfillment" - Massive existential frustration

Logo-test reliability as used in the research was high reflecting a Cronbach $\alpha=0.83$.

\section{Results}

All statistical analyses were carried out using the SPSS software (Reliability tests and Pearson's correlation tests). In examining the results, there was a significant correlation between the level of satisfaction with social support from relatives of the dying client and the degree of meaningfulness of his or her life. Based on the results given in Table 1 , the value of the correlation coefficient $r=-0.481$ is significant. This means that the more the client is satisfied with his or her social support from relatives, the higher is the meaningfulness of his or her life.

The interviews indicated that social support for dying clients is most frequently provided by family members, visits from children (son, daughter), and followed by friends and hospice staff. Most respondents were visited by their children - son (44\%), daughter (25\%), followed by friends (22\%). Only $9 \%$ felt the most social support from the staff and $6 \%$ did not feel any social support. Patients were least likely to be visited by their partners. A conclusion can be made that the decreased visits by partners is due to the fact that the average age of participants was almost 77, thus their partners either no longer lived or were also compromised by illness or old age.

Next, the correlation between the awareness of the client and the meaning of life was examined. Based on the results, a conclusion can be made that the better informed the client was during hospitalization, a higher purpose in life $(\mathrm{r}=-0.426$, sig. $=0.015, \mathrm{p}<0.05)$ was noted (Table 1). The research aimed to verify the level of awareness about nursing plan, medical treatment and its possible side effects, as well as socio-legal matters and the progress of care in general.

The last part of the research (Table 1) focused on the fulfillment of bio-psycho-socio-spiritual needs of the patient. It was found that the more satisfied the dying client was with the fulfillment of needs, the higher was the purpose in life $(r=-0.381$, sig. $=0.031, \mathrm{p}<0.05)$.

Another question examined in the study, focuses on which members of the staff are most frequently chosen by clients to discuss the meaning of life. According to their own statements, clients discuss the subject most frequently with a spiritual person - priest or nun (22\%) but also with a nurse $(16 \%)$ or a doctor $(15 \%)$. Some clients like to talk to anyone who shows a willingness to listen or offer advice (22\%). Some patients discuss the meaning of life with nobody (19\%) and other patients have no particular need to distinguish with whom they can openly talk and with whom it is better to remain reserved (6\%).

\section{Discussion}

The study suggests that the more satisfied the dying client was with social support from relatives, the higher was the degree of meaningfulness of life $(r=-0.481$; sig. $=0.005)$. The research shows that social support was most often provided by the son, whereas the husband/wife is the least present, either for health reasons or because the spouse is no longer alive ( 15 of the total of 32 clients were widowed

Table 1. Correlations.

\begin{tabular}{|c|c|c|c|c|c|}
\hline & & Social Support & Awareness & Logo-Test & $\begin{array}{l}\text { Satisfaction with } \\
\text { fulfillment of needs }\end{array}$ \\
\hline \multirow[t]{3}{*}{ Social Support } & Pearson Correlation & 1 & 0.153 & $-0.481 * *$ & -0.019 \\
\hline & Sig. (2-tailed) & . & 0.402 & 0.005 & 0,916 \\
\hline & $\mathrm{N}$ & 32 & 32 & 32 & 32 \\
\hline \multirow[t]{3}{*}{ Awareness } & Pearson Correlation & 0.153 & 1 & $-0.426^{*}$ & $0.583^{*}$ \\
\hline & Sig. (2-tailed) & 0.402 & . & 0.015 & 0.000 \\
\hline & $\mathrm{N}$ & 32 & 32 & 32 & 32 \\
\hline \multirow[t]{3}{*}{ Logo-Test } & Pearson Correlation & $-0.481^{*}$ & $-0.426^{*}$ & 1 & $-0.381^{*}$ \\
\hline & Sig. (2-tailed) & 0.005 & 0.015 & . & 0.031 \\
\hline & $\mathrm{N}$ & 32 & 32 & 32 & 32 \\
\hline \multirow{3}{*}{$\begin{array}{l}\text { Satisfaction with fulfillment of } \\
\text { needs }\end{array}$} & Pearson Correlation & -0.019 & $0.583 * *$ & $-0.381^{*}$ & 1 \\
\hline & Sig. (2-tailed) & 0.916 & 0.000 & 0.031 & . \\
\hline & $\mathrm{N}$ & 32 & 32 & 32 & 32 \\
\hline
\end{tabular}


at the time the study). A similar research project conducted by Dobríková [17] suggests that social support is most often provided by the daughter. Results of another research study conducted by Drageset et al. [18] supports the notion that social support of the dying is a means of improving the final stages of life. Herth [19], for instance, identified that factors arising from social relationships, such as loneliness and isolation are obstacles in dealing with illness and increasing hope in patients. These factors further increase the effect of fear and suffering on the dying, and thus hinder peaceful death. Herth [19] also describes four sources of hope: family, friends, health professionals and God or another higher being. The results of a national longitudinal research study among older persons suggested that even the mere anticipation of social support increases the meaningfulness of human life [20]. Research has confirmed a significant correlation $(r=-0.35, \mathrm{p}<0.001)$ between the feelings of loneliness and sense of life. On the other hand, the lonelier a person felt, the lower was the perception of the meaning of life.

Another interesting finding is that the better the client is informed about their overall condition, treatment process and the issues concerning care, the higher the level of meaningfulness perceived $(\mathrm{r}=$ $-0.426, \mathrm{p}<0.05$ ). A dying person needs to feel that people immediately involved in their care see him/her as a human being until the very end; that the patient is also being kept sufficiently informed about aspects of care that affect every-day life. This is closely linked to the client's confidence in the people who provide care. The current research also focuses on the needs (biological, psychological, social and spiritual) of dying clients, whereby it has been confirmed that the more satisfied the client is with the fulfilment of personal needs, the higher the level of purpose in life $(\mathrm{r}=-0.381$; sig. $=0.031, \mathrm{p}<0.05)$. As for biological needs, clients in the study found it very important to be "in no pain" (it must be said that this was an important point for all respondents, that is for $100 \%$ of clients), which in fact is the goal of hospice care - to guarantee that the client will not suffer unbearable pain. In the area of the clients' social needs, the need for the presence of a loving person who can be talked to, or can assist with personal hygiene, eating and such, was found to be very important. More importantly, it was crucial that family members are at hand in the time of need.

\section{Limitations}

This study has several limitations. There are some limits to generalizability because there are some specific themes which we can identify as a post-communist country-appropriate specific in the Slovak Repbulic and cannot be generalized to other societies and cultures. There is a small sample size. Many exclusion criteria were present and used to eliminate subjects. However, we consider the obtained results as important because there are few research studies on this topic in Slovak Republic and other countries and research in palliative care directly with patient involvement is very essential to delivering high-quality palliative care. Finally, the patients were in hospital based hospice care units and other hospice settings may not reveal similar results.

\section{Conclusion}

Caring for the dying person is not just about attending to physical symptoms and controlling pain. The meaning of life must be recognized and discussed as part of the treatment process. People have biological, psychological, social, and spiritual needs. Including family members as part of the treatment team is very important. Helping patients to decrease their negative feelings and respecting their dignity is crucial to meeting the needs of the patient. During the process of dying, social support, communication, and involvement of family increases the level of satisfaction with care which in turn influences a person's search for meaningfulness of life. Social support is conducted with awareness, satisfaction and meaningfulness of life. Consequently, treatment care teams need to pay attention to bio-psycho-social-spiritual needs of the patient when assessing total patient care and level of perceived satisfaction.

\section{Conflict of interest}

The authors declared no potential conflicts of interest with respect to the research, authorship, and/or publication of this article.

\section{Acknowledgement}

This study was conducted as part of the project "The Identity of Social Work in the Context of Slovakia [APVV-0524-12]" funded by the Slovak Research and Development Agency. The authors received financial support from the Slovak Research and Development Agency for the research, authorship, and/or publication of this article.

\section{References}

1. World Health Organization (2014) WHO Definition of Palliative Care

2. Sherbourne CD, Stewart AL (1991) The MOS social support survey. Soc Sci Med 32: 705-714. [Crossref]

3. Helgeson VS (2003) Social support and quality of life. Qual Life Res 12: 25-31. [Crossref]

4. Schwartz C, Frohner R (2005) Contribution of demographic, medical, and social support variables in predicting the mental health dimension of quality of life among people with multiple sclerosis. Health \& Social Work 30: 203-212.

5. Prince-Paul M (2008) Understanding the meaning of social well-being at the end of life. Oncol Nurs Forum 35: 365-371. [Crossref]

6. Mikulincer M, Florian V, Hirschberger G (2003) The existential function of close relationships: introducing death into the science of love. Personality and Social Psychology Review 7: 20-40.

7. Cassel EJ (1982) The nature of suffering and the goals of medicine. N Engl J Med 306 639-645. [Crossref]

8. Kissane DW, Clarke DM, Street AF (2001) Demoralization syndrome--a relevant psychiatric diagnosis for palliative care. J Palliat Care 17: 12-21. [Crossref]

9. Yalom I (1980) Existential Psychotherapy. New York: Basic Books, Inc.

10. Bolmsjö I, Hermerén G, Ingvar C (2002) Meeting existential needs in palliative carewho, when, and why? J Palliat Care 18: 185-191. [Crossref]

11. Frankl VE (2010) Vôla k zmyslu (The Will to Meaning). Bratislava, Slovak Republic: Lúc, 279s.

12. Blinderman CD, Cherny NI (2005) Existential issues do not necessarily result in existential suffering: lessons from cancer patients in Israel. Palliative Medicine 19: 371-380.

13. Moadel A, Morgan C, Fatone A (1999) Seeking meaning and hope: self-reported spiritual and existential needs among an ethnically-diverse cancer patient population. Psycho- Oncology Journal 8: 378-85.

14. Balcar K (1995) Standardizace dotazníku Logo-test "na vzorku studijných ceských vysokých škol. Ceskoslovenská Psychologie 39: 400-405.

15. McLafferty CL (1997) Spirituality in three theories of psychology: A qualitative study (Doctoral dissertation) University of Virginia. Dissertation Abstracts International 58: 1567.

16. Thege K, Martos T, Bachner YG, Talma Kushnir T (2010) Development and psychometric evaluation of a revised measure of meaning in life: The Logo-Test- R. Studia Psychologica 52: 155-163.

17. Dobríková P (2010) Quality of life in incurable patients. Studia Psychologica 52: 155- 163.

18. Drageset J, Eide GE, Nygaard HA, Bondevik M, Nortvedt MW, et al. (2009) The impact of social support and sense of coherence on health-related quality of life among nursing home residents-a questionnaire survey in Bergen, Norway. International Journal of Nursing Studies 46: 65-75. 
19. Herth K (1990) Fostering hope in terminally-ill people. J Adv Nurs 15: 1250-1259. [Crossref]
20. Krause N (2007) Longitudinal study of social support and meaning in life. Psychol Aging 22: 456-469. [Crossref]

Copyright: ( 2016 Dobríková P. This is an open-access article distributed under the terms of the Creative Commons Attribution License, which permits unrestricted use, distribution, and reproduction in any medium, provided the original author and source are credited. 